\title{
Minorities' Diminished Returns of Educational Attainment on Hospitalization Risk: National Health Interview Survey (NHIS)
}

\author{
Shervin Assari ${ }^{1 *}$, Mohsen Bazargan ${ }^{1,2}$ \\ 'Department of Family Medicine, Charles R. Drew University of Medicine and Science, Los Angeles, USA \\ ${ }^{2}$ Department of Family Medicine, UCLA, Los Angeles, USA
}

*Corresponding Author: Shervin Assari, MD, MPH, Department of Family Medicine, Charles R. Drew University of Medicine and Science, Los Angeles, USA. Tel: +1-734-232-0445, Fax: +1-734-615-8739, Email: assari@umich.edu

Received June 17, 2019; Accepted September 2, 2019; Online Published September 18, 2019

\begin{abstract}
Background: As suggested by the Minorities' Diminished Returns (MDRs) theory, educational attainment shows a weaker protective effect for racial and ethnic minority groups compared to non-Hispanic Whites. This pattern, however, is never shown for hospitalization risk.

Objectives: This cross-sectional study explored racial and ethnic variations in the association between educational attainment and hospitalization in the United States.

Methods: Data came from the National Health Interview Survey (NHIS 2015). The total sample was 28,959 American adults. Independent variable was educational attainment. The main outcome was hospitalization during the last 12 months. Age, gender, employment, marital status, region, obesity, and number of cardiovascular conditions were covariates. Race and ethnicity were the effect modifiers. Logistic regression models were utilized to analyze the data.

Results: From all participants, $16.2 \%$ were Black and $11.6 \%$ were Hispanic with a mean age of 51 years. Overall, higher education levels were associated with lower odds of hospitalization, independent of all confounders. Educational attainment showed significant interactions with race (odds ratio $[\mathrm{OR}]=1.04,95 \% \mathrm{Cl}=1.01-1.08)$ and ethnicity $(\mathrm{OR}=1.04,95 \% \mathrm{Cl}=1.01-1.07)$ on hospitalization, indicating smaller protective effects of educational attainment on hospitalization of Hispanics and Blacks than nonHispanic Whites.

Conclusion: The protective effects of educational attainment on population health are smaller for Blacks and Hispanics compared to non-Hispanic Whites. To prevent health disparities, the diminished returns of educational attainment should be minimized for racial and ethnic minorities. To do so, there is a need for innovative and bold economic, public, and social policies that do not limit themselves to equalizing socioeconomic status, but also help minorities leverage their available resources and gain tangible outcomes.
\end{abstract}

Keywords: Race, Ethnicity, Educational Attainment, Minorities' Diminished Returns, Socioeconomic Status, Hospitalization

\section{Background}

High socioeconomic status (SES), particularly educational attainment, is protective against undesired health outcomes. ${ }^{1-3}$ Individuals with high educational attainment are less likely to develop cardiovascular disease (CVD), which is the main cause of morbidity ${ }^{1,4-10}$ Racial and ethnic inequalities in health are due, in part, to racial and ethnic inequalities in SES and educational attainment. ${ }^{11}$

However, the protective effects of SES indicators on health outcomes differ among populations. ${ }^{12-15}$ The effects of SES indicators, particularly educational attainment, on CVDs risk are also shown to vary across populations. ${ }^{13,14}$ According to the Minorities' Diminished Returns (MDRs) theory, ${ }^{16,17}$ SES indicators, particularly educational attainment, are less protective for racial and ethnic minority groups than for majority groups. ${ }^{18}$ Most of the literature on this issue, however, has focused on the effects of SES on health outcomes for Blacks ${ }^{18-20}$ and Hispanics ${ }^{21,22}$ compared to Whites. For example, various studies have shown that SES indicators show larger effects on smoking ${ }^{23}$ and drinking ${ }^{21}$ for non-Hispanic Whites than for Hispanics or Blacks.

Very limited knowledge, however, exists on MDRs on the risk of hospitalization risk. One study has focused on MDRs on the use of healthcare services. In a study, family SES better reduced unmet health needs for Whites than for Hispanics and non-Hispanics. ${ }^{24}$ Thus, it seems that the very same MDRs that are established on health for Hispanics

Copyright (C) 2019 The Author(s). This is an open-access article distributed under the terms of the Creative Commons Attribution License (http:// creativecommons.org/licenses/by/4.0), which permits unrestricted use, distribution, and reproduction in any medium, provided the original work is properly cited. 
and Blacks ${ }^{18-20}$ may also be relevant to health service use.

\section{Objective}

This study explored racial and ethnic variations in the effects of educational attainment on the odds of hospitalization over the past 12 months among Americans in the United States. It was hypothesized that high educational attainment would be associated with lower hospitalization risk; however, this effect would be smaller for Hispanics and Blacks than for non-Hispanic Whites.

\section{Methods}

\subsection{Design and Settings}

The National Health Interview Survey (NHIS) of 2015 was used for this study. The NHIS is the primary source of information on the health and wellbeing of American adults. The sample was limited to the civilian noninstitutionalized population of the United States. Data was collected by the National Center for Health Statistics (NCHS), CDC.

\subsection{Data Collection}

The U.S. Census Bureau acts as the data collection agent for the NHIS under a contractual agreement between the two organizations. Interviews for the NHIS are conducted face-to-face and in the participants' residences. The interviews are sometimes followed with and, on rare occasions, replaced by telephone interviews. The NHIS data is collected continuously throughout each year. Data is collected on demographic factors, socioeconomic characteristics, health behaviors, mental health, physical health, and utilization of healthcare facilities.

\subsection{Sample and Sampling}

The NHIS sampling and sample design are described elsewhere. The NHIS applies a multi-stage sampling strategy. Stage 1 of the sample design was the sampling of 428 primary sampling units (PSUs) drawn from 1,900 geographically defined PSUs, which may be a county, a small group of contiguous counties, or a metropolitan statistical area. All 50 US states and the District of Columbia have representative PSUs in the study.

The NHIS draws samples from households and noninstitutional group quarters such as college dormitories and has four main cores: (1) the Household Composition section, (2) the Family Core, (3) the Sample Child Core, and (4) the Sample Adult Core.

\subsection{Inclusion and Exclusion Criteria}

Individuals were eligible for participation if they were adults (age 18 years or more) and US residents. Those institutionalized in a correctional or healthcare setting were ineligible to participate.

\subsection{Measures}

3.5.1. Predictor

Educational Attainment. Educational attainment was a continuous variable ranging from 0 to 21 . Participants were asked about the number of years of schooling they had. A higher score reflected a higher number of years of schooling.

\subsubsection{Moderators}

Race and Ethnicity. All participants self-identified their race and ethnicity. Races were Blacks/African Americans $=1$ and Whites $=0$. Ethnicity was a dichotomous variable: Hispanics $=1$, Non-Hispanics $=0$.

\subsubsection{Covariates}

Demographic Factors. Demographic data included participant age and gender. Age was continuous, but gender was dichotomous: males $=1$, females $=0$.

Marital status. A dichotomous variable was used for marital status: 1 married, 0 any other status.

Employment. A single item was used to measure employment over the prior week. This variable was a binary variable: employed $=1$, unemployed $=0$.

Region. Region was a four-level categorical variable coded as (1) Northeast, (2) Midwest, (3) South, and (4) West. This variable was treated as a nominal variable with West as the reference group.

Obesity. The current study defined obesity as a body mass index (BMI) equal to or larger than $30 \mathrm{~kg} / \mathrm{m}^{2} .{ }^{25}$ This was based on data on individuals' self-reported weight and height.

Number of CVDs. Number of CVDs was measured using the self-reported history of doctor-diagnosed CVDs. Participants reported if a doctor had told them that they have 1) diabetes, 2) hypertension, 3) high cholesterol, 4) coronary heart disease, 5) angina pectoris, 6) heart attack, 7) heart condition/disease, or 8) stroke. A sum CVD score ranging from 0 to 8 was calculated, and a higher score indicated more CVDs.

\subsubsection{Dependent Variable}

Hospitalization. Participants were asked if they have been hospitalized overnight during the prior 12 months. Possible answers were yes and no.

\subsection{Statistical Analysis}

Data was analyzed using SPSS 23.0 (IBM Inc, NY, USA). For descriptive statistics, mean and proportion (frequencies) were reported. Two survey logistic regression models were used for multivariable analysis. In these models, educational attainment was used as the independent variable, hospitalization as the dependent variable, demographic factors, marital status, employment, region, obesity, and number of CVDs as covariates, and race and ethnicity as the focal moderators. Both logistic regression models were estimated in the pooled sample that included Blacks, Hispanics, and non-Hispanic Whites. Model 1 did not include race or ethnicity by educational attainment interaction terms, but Model 2 did. Cox \& Snell R Square and Nagelkerke R Square were used to compare the fit of 
Model 1 and Model 2. Adjusted Odds Ratios (ORs), 95\% Confidence Intervals (CI), and $P$ values were reported.

\section{Results}

\subsection{Descriptive Statistics}

A total number of 28,959 individuals entered this analysis. Table 1 summarizes the descriptive statistics of the participants overall. On average, the participants were 51 years old. Most of the sample were White $(83.8 \%)$ and non-Hispanic $(88.4 \%)$. Most participants were female (55.3\%). From all participants, 54.2\% were employed, $43.4 \%$ were married, $34.1 \%$ were obese, and $10.1 \%$ had been hospitalized during the 12 months prior to the study (Table 1).

\subsection{Logistic Regressions}

Table 2 presents the results of 2 pooled sample logistic regression models. Both models were statistically significant at the 0.001 level. Cox \& Snell R Square for Model 1 and Model 2 were 0.057 and 0.058. Nagelkerke $\mathrm{R}$ square were 0.120 and 0.121 for Model 1 and Model 2, respectively. These fit statistics suggest that Model

Table 1. Descriptive Statistics of the Participants Overall

\begin{tabular}{|c|c|c|}
\hline & No. & $\%$ \\
\hline \multicolumn{3}{|l|}{ Race } \\
\hline White & 24270 & 83.8 \\
\hline Black & 4689 & 16.2 \\
\hline \multicolumn{3}{|l|}{ Ethnicity } \\
\hline Non-Hispanic & 25609 & 88.4 \\
\hline Hispanic & 3350 & 11.6 \\
\hline \multicolumn{3}{|l|}{ Gender } \\
\hline Female & 16017 & 55.3 \\
\hline Male & 12942 & 44.7 \\
\hline \multicolumn{3}{|l|}{ Married } \\
\hline Other & 16397 & 56.6 \\
\hline Married & 12562 & 43.4 \\
\hline \multicolumn{3}{|l|}{ Employed } \\
\hline Unemployed & 13264 & 45.8 \\
\hline Employed & 15695 & 54.2 \\
\hline \multicolumn{3}{|l|}{ Region } \\
\hline Northeast & 4884 & 16.9 \\
\hline Midwest & 6593 & 22.8 \\
\hline South & 10633 & 36.7 \\
\hline West & 6849 & 23.7 \\
\hline \multicolumn{3}{|l|}{ Obese (BMI > 30) } \\
\hline No & 19074 & 65.9 \\
\hline Yes & 9885 & 34.1 \\
\hline \multicolumn{3}{|l|}{ Hospitalized } \\
\hline No & 26032 & 89.9 \\
\hline \multirow[t]{2}{*}{ Yes } & 2927 & 10.1 \\
\hline & Mean & SD \\
\hline Age $(y)$ & 50.91 & 18.41 \\
\hline Education (y) & 15.19 & 3.14 \\
\hline Cardiovascular diseases (n) & 1.00 & 1.31 \\
\hline
\end{tabular}

2 showed statistically better fit compared to Model 1 . Model 1 only included the main effects; however, model 2 , also included two interaction terms between race and ethnicity with educational attainment. Model 1 showed that high educational attainment had a protective effect against hospitalization (odds ratio $[\mathrm{OR}]=0.99,95 \% \mathrm{CI}$ $=0.97-1.00$ ) above and beyond our covariates. Model 2 showed significant interactions between the effects of race $(\mathrm{OR}=1.04,95 \% \mathrm{CI}=1.01-1.08)$ and ethnicity (OR $=1.04,95 \% \mathrm{CI}=1.01-1.07)$ and educational attainment on hospitalization, suggesting that the inverse association between educational attainment and odds of hospitalization is significantly smaller for Blacks and Hispanics than for non-Hispanic Whites (Table 2).

\section{Discussion}

Higher educational attainment was inversely associated with hospitalization in the pooled sample; however, this effect was smaller for Blacks and Hispanics than for nonHispanic Whites.

It was found that, as educational attainment increases, people's risk of hospitalization decreases. This is, however, less true for Hispanic and Black Americans than for nonHispanic Whites. This is a replication of MDRs ${ }^{16,17}$ of the educational attainment in terms of hospitalization.

The results are in line with other observations that SES indicators, particularly educational attainment, generate less than expected health outcomes for marginalized groups than for mainstream ones. Similar patterns are shown for Blacks, ${ }^{26-29}$ Hispanics, ${ }^{21-23,30}$ and sexual minorities, ${ }^{31,32}$ meaning that minority status, regardless of whether it is based on race, ${ }^{33}$ ethnicity, ${ }^{21,22}$ or sexual orientation, ${ }^{31}$ reduces the health gains of SES resources. ${ }^{33}$

It is not only educational attainment, ${ }^{34}$ but any SES indicator that generates less health for Blacks and Hispanics than for non-Hispanic Whites. The very same patterns are shown for income, ${ }^{19}$ occupation, ${ }^{35}$ and marital status. ${ }^{36}$ The effects of education and income on chronic medical conditions such as CVDs, ${ }^{19}$ asthma, ${ }^{37}$ obesity, ${ }^{18,38}$ and $\mathrm{ADHD}^{20}$ are also shown to be smaller for Hispanics and Blacks than for non-Hispanic Whites. Finally, education and other SES indicators have a larger effect on reducing mental health problems such as depression, ${ }^{39-41}$ suicide, ${ }^{41}$ and anxiety for non-Hispanic Whites than Blacks. ${ }^{36}$ Education also better reduces the risk of mortality for Blacks than for Whites. ${ }^{35}$ None of these patterns have been previously shown for Hispanics compared to nonHispanics. The contribution of this study is to extend this literature to hospitalization as the outcome to both racial and ethnic groups.

The systemic nature of the MDRs suggests that it is the society that differentially rewards and differently treats racial and ethnic groups. ${ }^{16,17}$ This study argues that it is probably the marginalization processes that reduce full participation in society, and it is the social structure and function that do not allow non-White groups to fully leverage their human and economic resources and turn 
Table 2. Association Between Educational Attainment and Hospitalization in the Pooled Sample

\begin{tabular}{|c|c|c|c|c|c|c|c|c|c|c|}
\hline & \multicolumn{5}{|c|}{ Model 1 (Main Effects) } & \multicolumn{5}{|c|}{ Model 2 (M1 + Interactions) } \\
\hline & b & SE & B & $95 \% \mathrm{Cl}$ & $\boldsymbol{P}$ & b & SE & B & $95 \% \mathrm{Cl}$ & $P$ \\
\hline Race (Black) & 0.00 & 0.06 & 1.00 & $0.90-1.12$ & .943 & -0.60 & 0.25 & 0.55 & $0.33-0.90$ & 0.017 \\
\hline Ethnicity (Hispanic) & -0.07 & 0.07 & 0.93 & $0.81-1.08$ & .342 & -0.59 & 0.23 & 0.55 & $0.35-0.87$ & 0.010 \\
\hline Gender (Male) & -0.23 & 0.04 & 0.80 & $0.73-0.87$ & $<.001$ & -0.23 & 0.04 & 0.80 & $0.73-0.87$ & $<0.001$ \\
\hline Age $(y)$ & 0.00 & 0.00 & 1.00 & $0.99-1.00$ & .011 & 0.00 & 0.00 & 1.00 & $0.99-1.00$ & 0.013 \\
\hline Married & -0.13 & 0.04 & 0.88 & $0.81-0.96$ & .003 & -0.12 & 0.04 & 0.88 & $0.81-0.96$ & 0.005 \\
\hline Employed (last week) & -0.88 & 0.05 & 0.42 & $0.38-0.46$ & $<.001$ & -0.88 & 0.05 & 0.42 & $0.38-0.46$ & $<0.001$ \\
\hline \multicolumn{11}{|l|}{ Region } \\
\hline Northeast & 0.12 & 0.07 & 1.13 & $0.99-1.28$ & .080 & 0.12 & 0.07 & 1.12 & $0.99-1.28$ & 0.081 \\
\hline Midwest & 0.14 & 0.06 & 1.15 & $1.02-1.30$ & .022 & 0.14 & 0.06 & 1.15 & $1.01-1.29$ & 0.030 \\
\hline South & 0.07 & 0.06 & 1.07 & $0.95-1.20$ & .247 & 0.06 & 0.06 & 1.06 & $0.95-1.19$ & 0.292 \\
\hline Obesity (BMI > 30) & 0.05 & 0.04 & 1.06 & $0.97-1.15$ & .217 & 0.05 & 0.04 & 1.05 & $0.97-1.15$ & 0.241 \\
\hline Cardiovascular diseases (n) & 0.38 & 0.02 & 1.46 & $1.41-1.50$ & $<.001$ & 0.38 & 0.02 & 1.46 & $1.41-1.50$ & $<0.001$ \\
\hline Educational Attainment (y) & -0.01 & 0.01 & 0.99 & $0.97-1.00$ & .042 & -0.03 & 0.01 & 0.97 & 0.95-0.99 & $<0.001$ \\
\hline Ethnicity (Hispanic) × Educational Attainment $(y)$ & & & & & $<.001$ & 0.04 & 0.02 & 1.04 & $1.01-1.08$ & 0.014 \\
\hline Race (Black) × Educational Attainment (y) & & & & & & 0.04 & 0.02 & 1.04 & $1.01-1.07$ & 0.018 \\
\hline Constant & -1.89 & 0.14 & 0.15 & & & -1.65 & 0.16 & 0.19 & & $<0.001$ \\
\hline
\end{tabular}

them into the highest levels of tangible outcomes. ${ }^{16,17}$

\subsection{Future Research}

There is a need to study the contextual, economic, and behavioral mediators of MDRs of educational attainment on the risk of hospitalization. One potential mechanism for this observation is through $\mathrm{CVDs}^{19}$ as well as asthma, ${ }^{37}$ hypertension, ${ }^{30}$ obesity, ${ }^{18,38}$ depression, ${ }^{39-41}$ and suicidality. ${ }^{42}$ Another mechanism that may increase the risk of hospitalization in high SES Blacks and Hispanics is high risk behavior such as poor diet, ${ }^{43}$ poor exercise, ${ }^{27}$ smoking, ${ }^{23,44}$ and alcohol consumption. ${ }^{21}$ High SES Blacks and Hispanics are also exposed to higher levels of stress ${ }^{41,45-47}$ and more second-hand smoke. ${ }^{48}$ Research on public and health policies that reduce the MDRs of educational attainment are also needed. Future research should attempt to replicate and validate these findings in other contexts, settings, and age groups.

\subsection{Limitations}

This study has some methodological, conceptual, and statistical limitations. With a cross-sectional design, no causal inferences are drawn between educational attainment and risk of hospitalization. The study could not include data on a wide range of potential covariates such as healthcare access and mental health. Other SES indicators, such as wealth and childhood SES indicators, should also be measured. All study variables were measured at the individual level. Structural factors such as availability and density of healthcare services as well as area-level SES and racial and ethnic compositions of the neighborhoods were not included in this study. Despite these limitations, this is the first of its kind to investigate the relevance of MDRs to the risk of hospitalization.

\section{Conclusion}

The protective effects of educational attainment on hospitalization seem to be smaller for Hispanics and Blacks compared to non-Hispanic Whites. To prevent racial and ethnic health disparities, policies and programs should go beyond equal access and focus on equal outcomes. For such a goal, policies that minimize diminished returns of SES for racial and ethnic minorities are needed. Innovative and bold economic, public, and social policies are needed for the members of racial and ethnic minorities to leverage their resources and gain tangible health outcomes. Researchers may also explore mediators and moderators of MDRs for Black and Hispanic people.

\section{Authors' Contributions}

SA analyzed the data and prepared the first draft of the paper. MB revised the manuscript. Both authors approved the final draft.

\section{Conflict of Interest Disclosures}

The authors declare that they have no conflicts of interest.

\section{Ethical Approval}

All participants provided written consent, and the ethics of the NHIS protocol was approved by the CDC Institutional 


\section{Research Highlights}

\section{What Is Already Known?}

High educational attainment is known to protect individuals against risk of hospitalization. This effect is mainly due to better health status of individuals with higher education compared to individuals with lower educational levels.

\section{What This Study Adds?}

The protective effects of educational attainment on reducing risk of hospitalization is not equal across racial and ethnic groups. In the United States, highly educated Blacks and Hispanics remain at high risk of hospitalization, probably because of structural racism.

Review Board (IRB). According to the NIH guidelines and the decision tool regarding human subject involvement in secondary analyses of existing data, the current study was found to be a "Not Human Subject Research". The definition and decision tool which were applied are available here: https://grants.nih.gov/policy/humansubjects/hs-decision. htm. This study was found to be exempt from the IRB approval process for human involvement in research by the CDU IRB.

\section{Funding}

Assari and Bazargan were supported by the Center for Medicare and Medicaid Services (CMS) Grant $1 \mathrm{H} 0 \mathrm{CMS} 331621$ as well as the NIH under Award 54MD008149, R25 MD007610,2U54MD007598, and U54 TR001627. Assari was also supported by D084526-03 and CA201415-02 NIH grants.

\section{References}

1. Marmot M. Social determinants of health inequalities. Lancet. 2005;365(9464):1099-1104. doi:10.1016/s01406736(05)71146-6.

2. Marmot M. The Status Syndrome: How Social Standing Affects Our Health and Longevity. London: Bloomsbury Press; 2004.

3. Marmot M. Economic and social determinants of disease. Bull World Health Organ. 2001;79(10):988-989.

4. Assari S, Moghani Lankarani M. Income Gradient in Renal Disease Mortality in the United States. Front Med (Lausanne). 2017;4:190. doi:10.3389/fmed.2017.00190

5. Moghani Lankarani M, Assari S. Diabetes, hypertension, obesity, and long-term risk of renal disease mortality: Racial and socioeconomic differences. J Diabetes Investig. 2017;8(4):590599. doi:10.1111/jdi.12618.

6. Remes H, Martikainen P, Valkonen T. Mortality inequalities by parental education among children and young adults in Finland 1990-2004. J Epidemiol Community Health. 2010;64(2):136141. doi:10.1136/jech.2008.082388.

7. Farmer MM, Ferraro KF. Are racial disparities in health conditional on socioeconomic status? Soc Sci Med. 2005;60(1):191-204. doi:10.1016/j.socscimed.2004.04.026.

8. Phelan JC, Link BG, Diez-Roux A, Kawachi I, Levin B. "Fundamental causes" of social inequalities in mortality: a test of the theory. J Health Soc Behav. 2004;45(3):265-285. doi:10.1177/002214650404500303.

9. Mackenbach JP, Bos V, Andersen O, et al. Widening socioeconomic inequalities in mortality in six Western European countries. Int J Epidemiol. 2003;32(5):830-837. doi:10.1093/ije/dyg209.

10. Kunst AE, Groenhof F, Mackenbach JP, Health EW Occupational class and cause specific mortality in middle aged men in 11 European countries: comparison of population based studies. EU Working Group on Socioeconomic Inequalities in Health. BMJ. 1998;316(7145):1636-1642. doi:10.1136/ bmj.316.7145.1636.

11. Assari S. Number of Chronic Medical Conditions Fully Mediates the Effects of Race on Mortality; 25-Year Follow-Up of a Nationally Representative Sample of Americans. J Racial Ethn Health Disparities. 2017;4(4):623-631. doi:10.1007/ s40615-016-0266-4.

12. Assari S. Cross-Country Differences in the Additive Effects of Socioeconomics, Health Behaviors and Medical Comorbidities on Disability among Older Adults with Heart Disease. J Tehran Heart Cent. 2015;10(1):24-33.

13. Assari S, Lankarani MM. Does Multi-morbidity Mediate the Effect of Socioeconomics on Self-rated Health? Cross-country Differences. Int J Prev Med. 2015;6:85. doi:10.4103/20087802.164413

14. Assari S. Cross-country variation in additive effects of socioeconomics, health behaviors, and comorbidities on subjective health of patients with diabetes. J Diabetes Metab Disord. 2014;13(1):36. doi:10.1186/2251-6581-13-36

15. Assari S, Moghani Lankarani R, Moghani Lankarani M. Crosscountry differences in the association between diabetes and disability. J Diabetes Metab Disord. 2014;13(1):3. doi:10.1186/2251-6581-13-3

16. Assari S. Health disparities due to diminished return among black Americans: Public policy solutions. Soc Issues Policy Rev. 2018;12(1):112-145. doi:10.1111/sipr.12042.

17. Assari S. Unequal Gain of Equal Resources across Racia Groups. Int J Health Policy Manag. 2018;7(1):1-9. doi:10.15171/ijhpm.2017.90.

18. Assari S. Family Income Reduces Risk of Obesity for White but Not Black Children. Children (Basel). 2018;5(6). doi:10.3390/ children5060073.

19. Assari S. The Benefits of Higher Income in Protecting against Chronic Medical Conditions Are Smaller for African Americans than Whites. Healthcare (Basel). 2018;6(1). doi:10.3390/ healthcare6010002.

20. Assari S, Caldwell CH. Family Income at Birth and Risk of Attention Deficit Hyperactivity Disorder at Age 15: Racial Differences. Children (Basel). 2019;6(1). doi:10.3390/ children6010010.

21. Assari S, Farokhnia M, Mistry R. Education Attainment and Alcohol Binge Drinking: Diminished Returns of Hispanics in Los Angeles. Behav Sci (Basel). 2019;9(1). doi:10.3390/ bs9010009.

22. Assari S. Socioeconomic Status and Self-Rated Oral Health Diminished Return among Hispanic Whites. Dent J (Basel). 2018;6(2). doi:10.3390/dj6020011.

23. Assari S, Mistry R. Diminished Return of Employment on Ever Smoking Among Hispanic Whites in Los Angeles. Health Equity. 2019;3(1):138-144. doi:10.1089/heq.2018.0070.

24. Assari S, Hani N. Household Income and children's unmet dental care need; Blacks' diminished return. Dent J. 2018;6:17. doi:10.3390/dj6020017.

25. Chakraborty BM, Chakraborty R. Sensitivity and specificity of body mass index as a definition of the obesity component of 
metabolic syndrome. Coll Antropol. 2007;31(4):943-947.

26. Assari S, Preiser B, Kelly M. Education and Income Predict Future Emotional Well-Being of Whites but Not Blacks: A Ten-Year Cohort. Brain Sci. 2018;8(7). doi:10.3390/brainsci8070122.

27. Assari S. Educational Attainment and Exercise Frequency in American Women; Blacks' Diminished Returns. Womens Health Bull. 2019;6(3). doi:10.5812/whb.87413.

28. Assari S, Caldwell $\mathrm{CH}$, Mincy R. Family Socioeconomic Status at Birth and Youth Impulsivity at Age 15; Blacks' Diminished Return. Children (Basel). 2018;5(5). doi:10.3390/ children5050058.

29. Assari S. Parental Educational Attainment and Academic Performance of American College Students; Blacks' Diminished Returns. J Health Econ Dev. 2019;1(1):21-31.

30. Assari S. Socioeconomic Determinants of Systolic Blood Pressure; Minorities' Diminished Returns. J Health Econ Dev. 2019;1(1):1-11.

31. Assari S. Education Attainment and Obesity: Differential Returns Based on Sexual Orientation. Behav Sci (Basel). 2019;9(2). doi:10.3390/bs9020016.

32. Assari S, Bazargan M. Educational Attainment and Subjective Health and Well-Being; Diminished Returns of Lesbian, Gay, and Bisexual Individuals. Behav Sci (Basel). 2019;9(9). doi:10.3390/bs9090090.

33. Assari S. Parental Educational Attainment and Mental WellBeing of College Students; Diminished Returns of Blacks. Brain Sci. 2018;8(11). doi:10.3390/brainsci8110193.

34. Assari S. Blacks' Diminished Return of Education Attainment on Subjective Health; Mediating Effect of Income. Brain Sci. 2018;8(9). doi:10.3390/brainsci8090176.

35. Assari S, Moghani Lankarani M. Race and Urbanity Alter the Protective Effect of Education but not Income on Mortality. Front Public Health. 2016;4:100. doi:10.3389/fpubh.2016.00100.

36. Assari S, Caldwell CH, Zimmerman MA. Family Structure and Subsequent Anxiety Symptoms; Minorities' Diminished Return. Brain Sci. 2018;8(6). doi:10.3390/brainsci8060097.

37. Assari S, Moghani Lankarani M. Poverty Status and Childhood Asthma in White and Black Families: National Survey of Children's Health. Healthcare (Basel). 2018;6(2). doi:10.3390/ healthcare6020062.

38. Assari S, Thomas A, Caldwell CH, Mincy RB. Blacks' Diminished Health Return of Family Structure and Socioeconomic Status;
15 Years of Follow-up of a National Urban Sample of Youth. J Urban Health. 2018;95(1):21-35. doi:10.1007/s11524-0170217-3.

39. Assari S. High Income Protects Whites but Not African Americans against Risk of Depression. Healthcare (Basel). 2018;6(2). doi:10.3390/healthcare6020037.

40. Assari S. Social Determinants of Depression: The Intersections of Race, Gender, and Socioeconomic Status. Brain Sci. 2017;7(12). doi:10.3390/brainsci7120156.

41. Assari S, Moghani Lankarani M, Caldwell CH. Does Discrimination Explain High Risk of Depression among HighIncome African American Men? Behav Sci (Basel). 2018;8(4). doi:10.3390/bs8040040.

42. Assari S, Schatten HT, Arias SA, Miller IW, Camargo CA, Boudreaux ED. Higher Educational Attainment is Associated with Lower Risk of a Future Suicide Attempt Among NonHispanic Whites but not Non-Hispanic Blacks. J Racial Ethn Health Disparities. 2019;6(5):1001-1010. doi:10.1007/ s40615-019-00601-z.

43. Assari S, Moghani Lankarani M. Educational attainment promotes fruit and vegetable intake for whites but not blacks. J. 2018;1(1):29-41. doi:10.3390/j1010005.

44. Assari S, Mistry R. Educational Attainment and Smoking Status in a National Sample of American Adults; Evidence for the Blacks' Diminished Return. Int J Environ Res Public Health. 2018;15(4). doi:10.3390/ijerph15040763.

45. Assari S. Race, Intergenerational Social Mobility and Stressful Life Events. Behav Sci (Basel). 2018;8(10). doi:10.3390/ bs8100086.

46. Assari S, Moghani Lankarani M. Workplace Racial Composition Explains High Perceived Discrimination of High Socioeconomic Status African American Men. Brain Sci. 2018;8(8). doi:10.3390/brainsci8080139.

47. Assari S. Does School Racial Composition Explain Why High Income Black Youth Perceive More Discrimination? A Gender Analysis. Brain Sci. 2018;8(8). doi:10.3390/brainsci8080140.

48. Assari S, Bazargan M. Unequal Effects of Educational Attainment on Workplace Exposure to Second-Hand Smoke by Race and Ethnicity; Minorities' Diminished Returns in the National Health Interview Survey (NHIS). J Med Res Innov. 2019;3(2). doi:10.32892/jmri.179. 